\title{
Comparing Powder Magnetization and Transport Critical Current of $\mathrm{Bi}, \mathrm{Pb}(2223)$ Tapes
}

\author{
M. Dhallé, D. C. van der Laan, H. J. N. van Eck, L. Vargas, B. ten Haken, H. H. J. ten Kate, U. P. Trociewitz, and \\ J. Schwartz, Senior Member, IEEE
}

\begin{abstract}
The magnetic field dependence of the critical current in $(\mathrm{Bi}, \mathrm{Pb})_{2} \mathrm{Sr}_{2} \mathrm{Ca}_{2} \mathrm{Cu}_{3} \mathrm{O}_{10+x}$ tapes is compared with the magnetization response of isolated grains extracted from the tapes. Special attention is paid to the low-field behavior. The goal of the experiment is to test the widely-used hypothesis that current paths in these tapes contain both weak- and strong- linked branches, which in low field act in parallel. The data agree with this hypothesis; at temperatures above $50 \mathrm{~K}$ the powder magnetization drops off exponentially from the self-field to the irreversibility field, while the transport and magnetization currents in the intact tapes show an extra low-field component. Below $50 \mathrm{~K}$ the powder behavior becomes less straightforward, but the parallel-path picture in the tapes still holds.
\end{abstract}

Index Terms-Bi,Pb(2223) tapes, critical current, high-temperature superconductors, weak links.

\section{INTRODUCTION}

$\mathbf{T}$ $\mathrm{HE}$ field dependence of the critical current in $(\mathrm{Bi}, \mathrm{Pb})_{2} \mathrm{Sr}_{2} \mathrm{Ca}_{2} \mathrm{Cu}_{3} \mathrm{O}_{10+x}(\mathrm{Bi}, \mathrm{Pb}(2223))$ powder-in-tube (PIT) tapes typically shows two distinct regimes. At low field it has a relatively sharp drop-off, which is reasonably well described by an inverse power-law relationship between magnetic field and current. At higher fields the current decay becomes more gradual, acquiring an exponential signature (Fig. 1).

Already early on these observations lead to the so-called parallel-path model. The temperature-dependent characteristic field describing the exponential decay was found to show only small variations from one $\mathrm{Bi}, \mathrm{Pb}(2223)$ tape to another, irrespective of its overall critical current $\left(I_{c}\right)$ [1]. Furthermore, by properly taking into account experimental differences, this high-field behavior is essentially identical to the magnetic response not only of the tapes, but also of $\mathrm{Bi}, \mathrm{Pb}(2223)$ powders prepared in different ways [2]. In such isolated grains the current is not hampered by intergranular boundaries, so that one can conclude that the exponential decay is related to a dissipation mechanism within the grains, and in all likelihood, to

Manuscript received August 5, 2002.

M. Dhallé, H. J. N. van Eck, L. Vargas, B. ten Haken, and H. H. J. ten Kate are with the Department Applied Physics at the University of Twente, Enschede, the Netherlands (e-mail: m.j.dhalle@tn.utwente.nl).

D. C. van der Laan was with the National High Magnetic Field Laboratory, Tallahassee, FL 32310 USA. He is now with University of Twente, Enschede, the Netherlands.

U. P. Trociewitz is with the National High Magnetic Field Laboratory,Tallahassee, FL 32310 USA.

J. Schwartz is with the National High Magnetic Field Laboratory and the Department of Mechanical Engineering at Florida State University, Tallahassee, FL 32310 USA.

Digital Object Identifier 10.1109/TASC.2003.812520

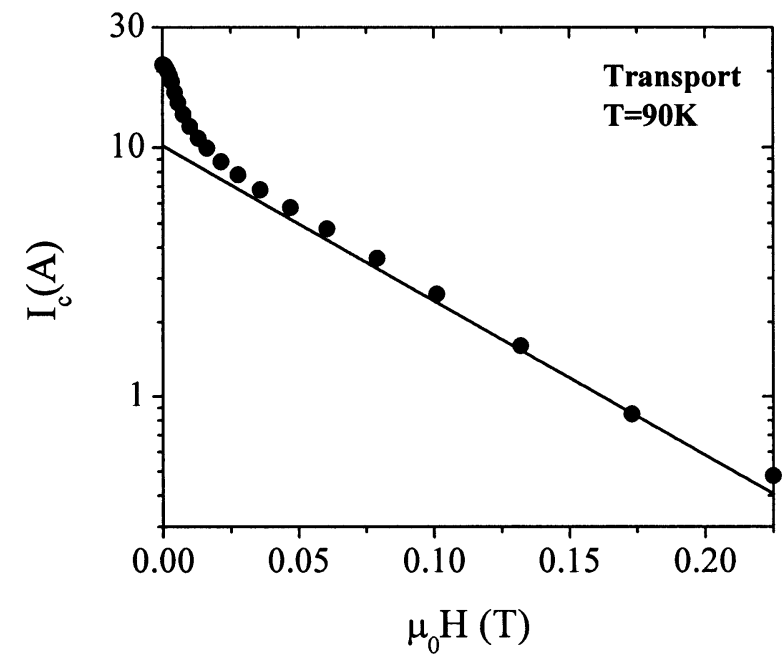

Fig. 1. Typical field dependence of the critical current in a $\mathrm{Bi}, \mathrm{Pb}(2223) \mathrm{PIT}$ tape. The symbols represent measured data, while the solid line indicates a pure exponential current-field relation.

magnetic flux motion. The fact that the same field dependence is found for transport currents indicates that macroscopic current paths exist in which flux motion within the grains, rather than dissipation at grain boundaries, is the current-limiting factor. These current paths were named "strong-linked" and significant microstructural effort has been invested in attempts to identify the type of grain boundary responsible for this dissipation-free transport [3], [4].

Unlike the $j_{c}(H)$ dependence in these strong-linked paths, the details of the relatively rapid low-field decay appear to be strongly sample dependent. From early experiences with bulk, untextured superconducting ceramics [5], it was concluded that the low-field dependence was due to the presence of additional current paths, in which grain boundaries do constitute weaklinks, so that this extra parallel current decayed rapidly with magnetic field.

This parallel-path model with a strong-linked backbone embedded within a weak-linked matrix has become widely accepted. It is useful in the sense that within this framework attempts can be made to quantify the relative contributions of both current paths and thus learn more about the differences between these "strong" and "weak" links in $\mathrm{Bi}, \mathrm{Pb}(2223)$ [6]. All these attempts hinge, however, on the assumption that $j_{c}(H)$ in the strong-linked current paths behaves exponentially throughout the entire field range, and that that any additional functional dependence in the overall $j_{c}(H)$ curves is only due 
to extra current paths. This assumption can be questioned in the light of the wealth of experiments on $(\mathrm{Bi}, \mathrm{Pb})_{2} \mathrm{Sr}_{2} \mathrm{CaCu}_{2} \mathrm{O}_{8+y}$ crystals [7], showing that flux motion in Bi-Sr-Ca-Cu-O ceramics can be quite complicated and can involve different vortex structures and pinning regimes, as well as geometrical complications.

The aim of this paper is to verify the validity of the parallel-path assumption through a careful comparison between powder magnetization and the overall $j_{c}(H)$ dependence of intact tapes. In this respect, it resembles earlier studies on $\mathrm{Bi}, \mathrm{Pb}(2223)$ tapes [1], but with two key differences. Firstly, whereas the earlier work focused on the high-field behavior, in order to bring out the similarities between the macroscopic critical current and the powder magnetization in this regime, our data are analyzed with special attention to the low-field regime. Secondly, to be certain that our powder data are free from any residual weak-link effects, we paid particular attention to the powder extraction and isolation procedure.

\section{EXPERIMENTAL}

\section{A. Sample Preparation and Measurements}

All samples were taken from one single batch of commercial 85-filament $\mathrm{Bi}, \mathrm{Pb}(2223) \mathrm{PIT}$ tape. The transport $I_{c}$ was measured with a four-point experiment in an $8 \mathrm{~T}$ magnet, using a controlled gas environment to stabilize the temperature [8]. All magnetization data were gathered in a commercial SQUID magnetometer equipped with a $6 \mathrm{~T}$ magnet.

To isolate individual $\mathrm{Bi}, \mathrm{Pb}(2223)$ grains, the external silver sheath was peeled away and the ceramic powder was scraped out, ground in mortar, and suspended in ethylene alcohol. After passing the suspension through filter paper with a $10 \mu \mathrm{m}$ pore size, the alcohol was allowed to evaporate and the remaining dust was gathered and mixed with Stycast 1266 epoxy. Fig. 2 shows that this procedure results in well-isolated grains.

\section{B. Data Analysis}

In the parallel path model, the weak- and strong-linked contributions are summed. To isolate the low-field contribution, we approximate the high-field behavior by a purely exponential $I_{c}(H)$ relationship and subtract it from the raw data. Some care must be taken, however; force-fitting an exponential curve introduces uncertainty, as the result depends on the choice of fitting interval. We avoid this problem using the field dependence of the macroscopic pinning force,

$$
F_{p}(H, T) \equiv H \times I_{c}(H, T) .
$$

As seen in Fig. 3(a), this pinning force has a well-defined maximum which can be used to extract a characteristic field $H_{p}(T)$ and force $F_{p, p e a k}(T)$. A perfectly exponential $I_{c}(H)$ dependence would be fully characterized by these two parameters:

$$
I_{c}(H, T)=\frac{F_{p, p e a k}(T)}{H_{p}(T)} \exp \left(1-\frac{H}{H_{p}(T)}\right)
$$



Fig. 2. SEM image of the $\mathrm{Bi}, \mathrm{Pb}(2223) /$ epoxy mixture. In this backscattered electron image the ceramic grains close to the epoxy surface show up as bright areas, the epoxy as the darker matrix.

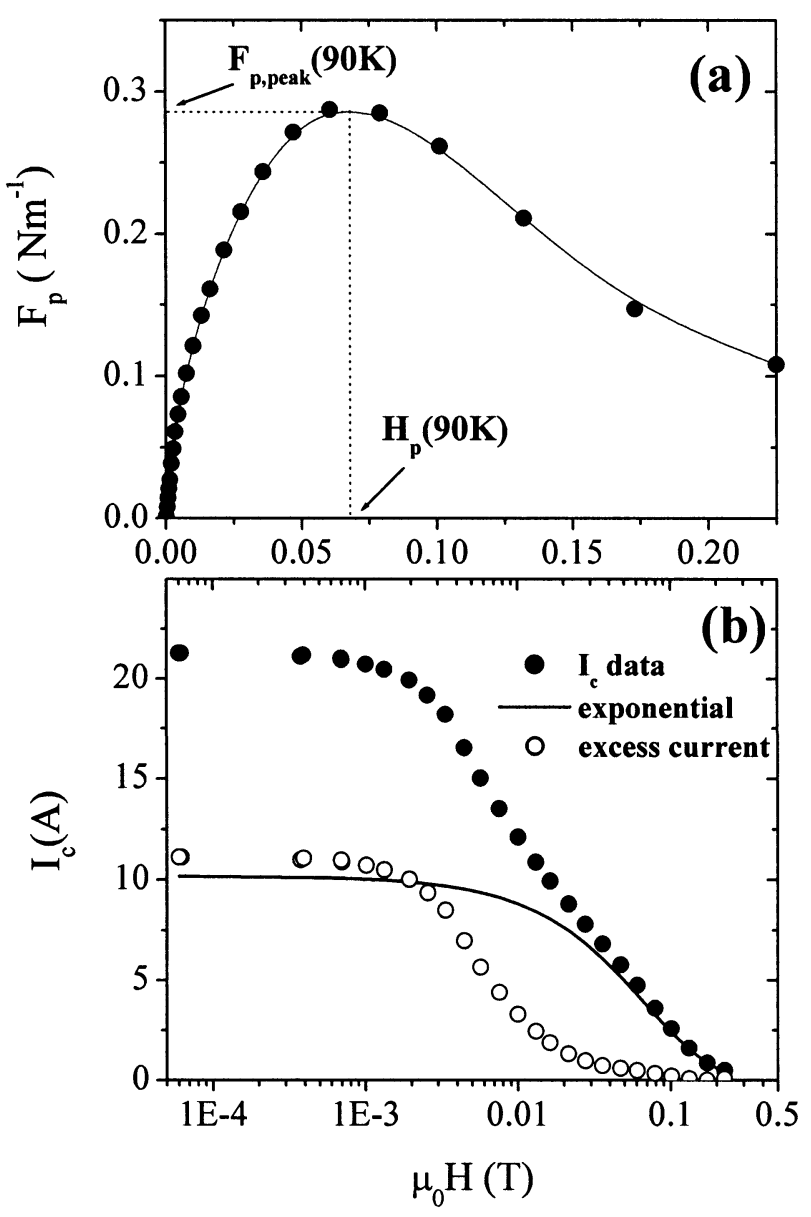

Fig. 3. (a) The field dependence of the macroscopic pinning force corresponding to the $I_{c}$ data shown in Fig. 1. The maximum in such curves can be used to extract a temperature dependent characteristic field and characteristic current. (b) The same data as in Fig. 1. The open symbols represent the excess current, obtained after subtracting the exponential field dependence given by (2).

In other words, for a perfectly exponential $I_{c}(H)$ dependence the peak in the macroscopic pinning force occurs exactly at the exponential decay field. 


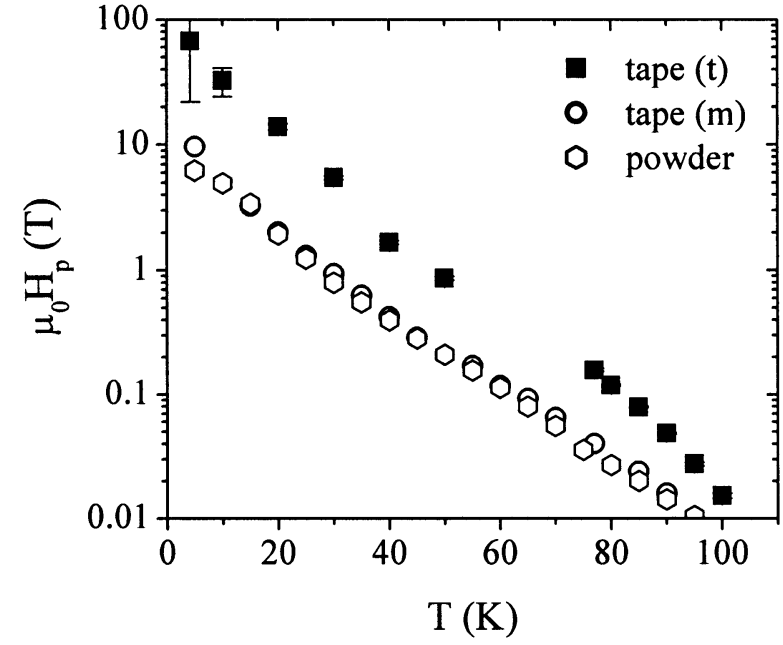

Fig. 4. The characteristic field determined by the peak in the pinning force, extracted from the transport $I_{c}$ data of the intact tape (solid squares), and from the irreversible magnetization for both tape (open squares) and powder (open hexagons).

\section{RESULTS}

The previously described analysis is applied to both transport and magnetization data. In the latter, we use the irreversible magnetization, which is proportional to the current density, instead of $I_{c}$. Fig. 4 shows the temperature dependence of the peak field $H_{p}(T)$ obtained from the transport and magnetization data, the latter for an intact tape and for the isolated powder. This figure confirms earlier results. First of all, the characteristic decay field is virtually identical for tape and powder magnetization, supporting the conclusion that intragranular flux motion constitutes the dominating dissipation mechanism in the tape at high field. Secondly, the transport data yield a characteristic decay field that is nearly ten times higher than that obtained by magnetization. This can be understood as a consequence of the different electric fields occurring in the experiments [1]. More importantly, the temperature dependence of $H_{p}$ is nearly the same for transport and magnetization. This indicates that also in transport experiments, where current necessarily flows on a macroscopic scale, it is flux motion that limits the overall current and not weak links.

Having confirmed the earlier conclusions, we focus on the low-field regime of $j_{c}(H)$. Even if our high-field data does not behave perfectly exponentially, we can still use the procedure described in Section II-B. to extract an excess current, using (2) to approximate the strong-linked contribution that is subtracted.

Fig. 5 shows the results of the analysis for the three types of measurements (transport, tape magnetization and powder magnetization). Each curve corresponds to a different temperature. To compare these three experiments more easily, the excess current or magnetization is plotted as a percentage of the overall current or magnetization.

Two conclusions are drawn immediately. Firstly, there is a clear correlation between transport and magnetization data, in amplitude as well as in temperature and field dependence of the excess current in the intact tape. Secondly, the powder data behave quantitatively differently; rather than a monotonous decrease with applied magnetic field, they show a maximum at

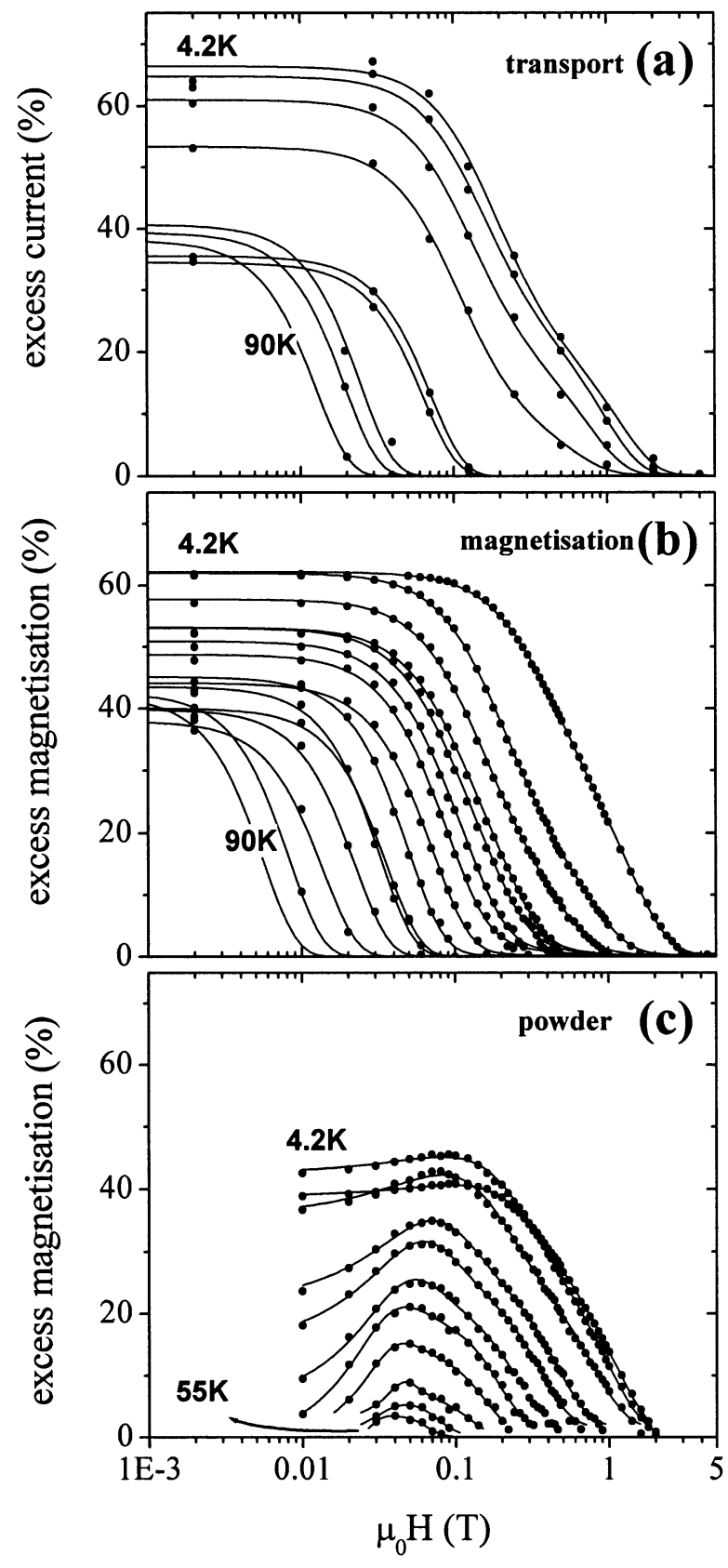

Fig. 5. (a) The excess transport current plotted against magnetic field at $T=$ $4.2,10,20, \ldots 50,77,80,85$ and $90 \mathrm{~K}$. (b) The excess magnetization of the intact tape plotted every five $\mathrm{K}$ between $T=5$ and $90 \mathrm{~K}$. (c) The excess magnetization of the powder sample, plotted every five $\mathrm{K}$ between $T=5$ and 55 $\mathrm{K}$. For ease of comparison, all data points are normalized to the total, measured current or magnetization.

nonzero magnetic field. Furthermore, the excess contribution to the powder magnetization disappears above $\sim 55 \mathrm{~K}$, i.e., above this temperature the irreversible powder magnetization behavior is essentially exponential, without additional low-field contributions.

These differences between the powder and the intact tape can also be seen in Fig. 6, which plots the maximum excess signal as a function of temperature. The excess powder signal decreases linearly with temperature to zero at $\sim 55 \mathrm{~K}$. In the same lowtemperature range, the excess tape signal also decreases linearly. 


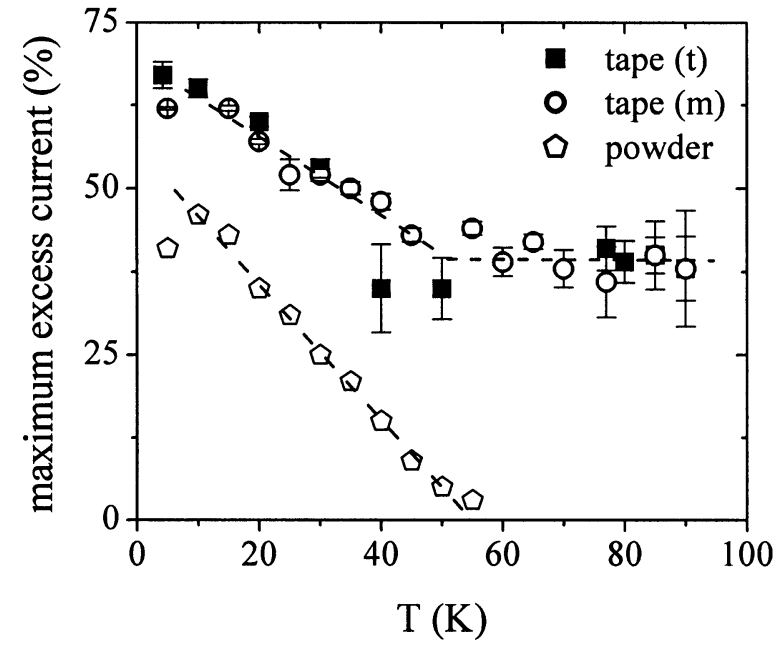

Fig. 6. The maximum amplitude of the excess currents shown in Fig. 5, plotted against temperature for tape transport and magnetization, as well as powder magnetization.

However, above $T \approx 55 \mathrm{~K}$ the low-field excess contribution becomes nearly temperature independent and for this tape stays at $\sim 40 \%$ of the overall current. In other words, once the field dependence of the irreversible powder magnetization becomes purely exponential, the excess component of the tape current becomes a constant fraction of the overall current.

\section{CONCLUSIONS}

We confirm the validity of the main assumption of the parallel-path model for $\mathrm{Bi}, \mathrm{Pb}(2223)$ tapes: the presence of two distinct and independent current contributions. Our data confirm this assumption in the sense that we indeed find an additional low-field current component in the intact tapes which is not present in the isolated powder. This conclusion agrees with the concept of a strong-linked backbone within a weak-linked matrix.
We also confirm earlier results that the current in the stronglinked backbone has the same field and temperature dependence as the magnetization currents in the isolated grains, and thus must be limited by intragranular flux motion, justifying the term "strong-linked."

As to the additional "weak-linked" current contribution, above $T=55 \mathrm{~K}$ its amplitude is a temperature-independent fraction of the strong-linked component. In other words, strong- and weak-linked currents have the same temperature dependence. This surprising conclusion merits further study, since it implies that weak-link dissipation and intragranular flux motion influence each other.

\section{REFERENCES}

[1] M. Dhallé, M. Cuthbert, M. D. Johnston, J. Everett, R. Flükiger, S. X. Dou, W. Goldacker, T. Beales, and A. D. Caplin, "Experimental assessment of the current-limiting mechanisms in BSCCO/Ag high-temperature superconducting tapes," Supercond. Sci. Technol., vol. 10, pp. 21-31, 1997.

[2] M. Dhallé, F. Marti, G. Grasso, A. Perin, J. C. Grivel, E. Walker, and R. Flükiger, "Critical currents in polycrystalline $(\mathrm{Bi}, \mathrm{Pb})_{2} \mathrm{Sr}_{2} \mathrm{Ca}_{2} \mathrm{Cu}_{3} \mathrm{O}_{10}$ systems: A comparative magnetization study," Physica C, vol. 282, pp. 1173-1174, 1997.

[3] B. Hensel, G. Grasso, and R. Flükiger, "Limits to the critical transport current in superconducting $(\mathrm{Bi}, \mathrm{Pb})_{2} \mathrm{Sr}_{2} \mathrm{Ca}_{2} \mathrm{Cu}_{3} \mathrm{O}_{10}$ silver-sheathed tapes-The railway-switch model," Phys. Rev. B, vol. 21, pp. 15 456-15 473, 1995.

[4] Y. H. Li, J. A. Kilner, M. Dhallé, A. D. Caplin, G. Grasso, and R. Flükiger, "Brick wall or railway switch-The role of low-angle ab-axis grain boundaries in the critical current of BSCCO tapes," Supercond. Sci. Technol., vol. 8, pp. 764-768.

[5] R. L. Peterson and J. W. Ekin, "Airy pattern, weak-link modeling of critical currents in high- $T_{c}$ superconductors," Physica $C$, vol. 156, pp. 325-333, 1989.

[6] Y. K. Huang, B. ten Haken, and H. H. J. ten Kate, "Critical current of high $T_{c}$ superconducting Bi2223/Ag tapes," Physica $C$, vol. 309, pp. 197-202, 1998.

[7] B. Khaykovich, E. Zeldov, D. Majer, T. W. Li, P. H. Kes, and M. Konczykowski, "Vortex-lattice phase transitions in $\mathrm{Bi}_{2} \mathrm{Sr}_{2} \mathrm{CaCu}_{2} \mathrm{O}_{8}$ crystals with different oxygen stoichiometry," Phys. Rev. Lett., vol. 76, pp. 2555-2558.

[8] B. ten Haken, A. Godeke, and H. H. J. ten Kate, "The strain dependence of the critical properties of $\mathrm{Nb}_{3}$ Sn conductors," J. Appl. Phys., vol. 85, pp. 3247-3253, 1999. 